\title{
POSTER
}

\section{Évaluation d'une OAM monobloc sur-mesure dans le traitement de SAHOS}

\section{Derrien $\mathrm{A}^{1}$, Boudaud-Gueguen $\mathrm{P}^{2}$, Mouallem $\mathrm{G}^{3}$, Praud $\mathrm{M}^{4}$, Humeau-Chapuis MP5 ${ }^{5}$, Kün-Darbois $\mathrm{JD}^{6}$, Boyer $\mathrm{L}^{7}$, Corre $\mathrm{P}^{8}$}

\section{1,2 3, 4, 6,8 - Service de Chirurgie Maxillo-Faciale et Stomatologie, Nantes, France}

5 - Le Confluent - Nouvelles Cliniques Nantaises ; Service de Pneumologie - CHU, Nantes, France

7 - Service de Pneumologie, Créteil, France

Introduction - Matériels

Les Orthèses d'Avancée Mandibulaire (OAM) sont des dispositifs permettant de traiter les Syndromes d'Apnées-Hypopnées Obstructives du Sommeil (SAHOS). Elles peuvent être utilisées en première intention chez les patients présentant des ronchopathies simples ou un SAHOS léger à modéré, sans comorbidité grave associée. On peut les utiliser en deuxième intention en cas d'intolérance ou d'échec des dispositifs de Pression Positive Continue (PPC). Enfin, on peut l'utiliser en complément de la PPC pour augmenter son efficacité.

Cette étude rétrospective a inclus 172 patients traités par OAM pour un SAHOS léger à sévère. Le but de cette étude est de démontrer l'efficacité d'une OAM monobloc sur-mesure dans le traitement du SAHOS.

\section{Méthodes}

Une mesure de l'Index d'Apnées Hypopnées (IAH) a été effectué avant, et 3 mois après la mise en place de l'OAM. On a réalisé également l'évaluation des effets secondaires, à long terme, du dispositif.

\section{Résultats}

Parmi les 172 patients inclus, on retrouvait $67 \%$ d'hommes, âgés en moyenne de 54,4 ans ; et $33 \%$ de femmes, âgées de 57,5 ans. Le recul était supérieur à 3 ans (environ 40 mois). Les IAH moyens des hommes et des femmes avant OAM étaient respectivement de 23,6 et 21,9, et après mise en place de l'OAM de 8,9 et 5,9. II existait une corrélation linéaire entre l'importance de l'IAH et sa correction par l'OAM. Aucune corrélation entre le sexe, l'Indice de Masse Corporelle (IMC) pré-OAM, l'âge, et la baisse de l'IAH n'a été observée. Nous avons recensé 18 échecs de l'OAM par insuffisance d'efficacité. Les effets secondaires retrouvés (5\%) étaient principalement des modifications occlusales, une intolérance du dispositif (douleur, hypersalivation, ou inconfort), et des bris d'OAM. Seuls 6 patients (3\%) ont arrêté l'OAM en raison d'effets secondaires.

\section{Discussion}

Les limites de cette étude résident dans son caractère rétrospectif qui a restreint le recueil de données sur l'observance ainsi que l'évaluation subjective de l'efficacité de l'OAM. 
Conclusion

Cette étude a démontré une efficacité de $89 \%$ de l'OAM monobloc dans le traitement du SAHOS, correspondant aux données les plus favorables de la littérature. Elle tendait même à montrer une efficacité dans les SAHOS avec IAH supérieur à 30. Les effets secondaires, à long terme, de I'OAM monobloc ne contre-indiquaient que rarement son utilisation.

Bettega G. Place de l'OAM dans le traitement des SAHOS de l'adulte. Recommandations de Bonnes Pratiques dans la prescription de l'OAM. 